\title{
Incidental finding of lipaemia retinalis on diabetic retinal screening
}

\author{
Punith Kempegowda1,2, Wentin Chen 3 , Eka Melson 11,2 , Annabelle Leong 4 , \\ Prashant Amrelia ${ }^{2}$ and Ateeq Syed ${ }^{2}$
}

'Institute of Metabolism and Systems Research, University of Birmingham, Birmingham, UK, 2University Hospitals Birmingham NHS Foundation Trust Birmingham, Birmingham, UK, ${ }^{3}$ Medical School, College of Medical and Dental Sciences, University of Birmingham, Birmingham, UK, and ${ }^{4}$ Health Education England West Midlands, Birmingham, UK

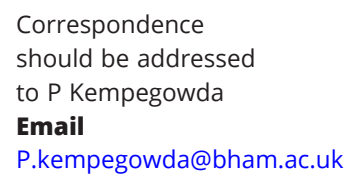

\section{Summary}

A 37-year-old female of South Asian origin was referred to our diabetes clinic for evaluation of an unusual finding during her retinal screening. Her retinal blood vessels appeared white in contrast to the normal pink-red colour. She had type I hyperlipidaemia, confirmed by genotype, and was recently diagnosed with diabetes, secondary to pancreatic insufficiency, for which she had suboptimal control and multiple hospitalisations with recurrent pancreatitis. On examination, she had multiple naevi on her skin; the rest of the examination was unremarkable. The patient did not report any visual disturbances and had intact visual acuity. Investigations showed raised total cholesterol (12.5 mmol/L) and triglycerides $(57.7 \mathrm{mmol} / \mathrm{L})$. Following evaluation, the patient was diagnosed with lipaemia retinalis, secondary to type I hyperlipidaemia. The patient was managed conservatively to reduce the cholesterol and triglyceride burdens. However, therapies with orlistat, statin, fibrates and cholestyramine failed. Only a prudent diet, omega-3 fish oil, medium-chain triglycerides oil and glycaemic control optimised with insulin showed some improvements in her lipid profile. Unfortunately, this led her to becoming fat-soluble vitamin deficient; hence, she was treated with appropriate supplementation. She was also recently started on treatment with volanesorsen. Following this, her lipid parameters improved and lipaemia retinalis resolved.

\section{Learning points:}

- Lipaemia retinalis is an uncommon incidental finding of type I hyperlipidaemia that may not affect vision.

- Management of associated dyslipidaemia is challenging with minimal response to conventional treatment.

- Increased awareness of lipaemia retinalis and specialist management is needed as part of regular patient monitoring and personalised management.

\section{Background}

Hyperlipidaemia is a metabolic syndrome defined as elevated serum triglyceride and cholesterol levels, with disturbances to low- and high-density lipoprotein concentrations. It can be primary, also called familial, or acquired and is common in patients with diabetes mellitus who present with hypertriglyceridaemia and/or hypercholesterolaemia $(1,2)$. Secondary causes of hyperlipidaemia include drugs, chronic alcohol abuse, uncontrolled diabetes mellitus, hypothyroidism and obesity $(1,3)$. It is the most common disorder of lipoprotein metabolism, affecting an estimated $10 \%$ of US children and adolescents (4). It increases the risk of 
atherosclerosis, with multisystem involvement including the gastrointestinal system, skin and eyes. Five subgroups of primary hyperlipidaemia have been defined based on the type of lipoproteins involved (Table 1) (5). Of these, types I, IV and $\mathrm{V}$ are characterised by hypertriglyceridaemia. Chylomicronaemia occurs with a triglyceride level $>25.9$ $\mathrm{mmol} / \mathrm{L}$ and is defined as chylomicronaemia syndrome associated with systemic signs and symptoms, such as eruptive xanthomas, recurrent episodes of abdominal pain, hypertriglyceridaemia and lipaemia retinalis $(6,7)$.

Lipaemia retinalis is a rare ocular manifestation and clinical sign of hypertriglyceridaemia and chylomicronaemia. It can occur in both children and adults (2). Its incidence correlates approximately with the level of plasma triglycerides; however, the key factor is the level of chylomicrons (6). It presents with creamy-white retinal blood vessels and a salmon-pink retina on fundoscopic examination, due to an increased chylomicron level in circulating retinal blood (6). Most cases do not affect visual acuity. Although described as rare, Brunzell and Bierman reported that lipaemia retinalis is underdiagnosed and occurs in $23 \%$ of patients with chylomicronaemia, which may be explained by the lack of routine fundoscopic examination (7). Herein, we describe a patient with grade III lipaemia retinalis and the complexities of the patient management.

\section{Case presentation}

A 37-year-old South Asian woman with a 19-year history of genotype-confirmed type I hyperlipidaemia was referred to our diabetes clinic from the NHS Diabetic Eye Screening Programme for evaluation of an unusual finding during her retinal screening. Her retinal blood vessels appeared white, in contrast to the normal pink-red colour (Fig. 1).

The patient was diagnosed with diabetes mellitus, secondary to pancreatic insufficiency due to chronic pancreatitis nearly a year prior to this index presentation, for which she was on metformin and long-acting insulin but had suboptimal control and multiple hospitalisations

Table 1 Fredrickson-WHO classification for hyperlipidaemia (5). The five subgroups of hyperlipoproteinaemia and their common alternative names, the molecular abnormalities involved, the specific lipoproteins that are significantly elevated, the associated symptoms, the appropriate evidence-based treatments and the relative occurrence rates of the subgroups are presented.

\begin{tabular}{l} 
Hyperlipoproteinaemia, \\
type/synonyms \\
\hline Type I \\
Familial \\
hyperchylomicronaemia \\
Familial APOC2 deficiency \\
Buerger-Gruetz syndrome \\
Type II \\
Familial \\
hypercholesterolaemia \\
Familial combined \\
hyperlipidaemia \\
Type III \\
Familial \\
dysbetalipoproteinaemia \\
Type IV \\
Familial \\
hypertriglyceridaemia \\
Type V \\
Combined hypertriglyceri- \\
daemia
\end{tabular}

Defect

$\downarrow L P L$

Altered APOC2

LPL inhibitor in blood

\begin{tabular}{l}
$\begin{array}{l}\text { Increased serum } \\
\text { lipoprotein }\end{array}$ \\
\hline Chylomicron
\end{tabular}

Symptoms

Pancreatitis, lipaemia retinalis, xanthomas, hepatosplenomegaly

$\frac{\text { Treatment }}{\text { Diet }} \frac{\text { Occurrence }}{\text { Rare }}$

Xanthelasma, arcus senilis, corneal arcus, tendon xanthomas

$\downarrow L P L$ receptor $\quad$ LDL
$\downarrow$ LDL receptor; LDL, VLDL
$\uparrow A P O B$

APOE2 ID synthesis defect

†VLDL VLDL

production, $\downarrow$ Elimination

$\uparrow V L D L$ production, $\downarrow$ LPL palmar xanthoma

Lipaemia retinalis, pancreatitis, xanthomas

VLDL, chylomicron
Bile acid sequestrants, statins, niacin Statins, niacin, fibrate

Most common

Tubo-eruptive xanthomas, Fibrate, statins

Fibrate, niacin, statins
Lipaemia retinalis, xanthomas

APOB, apoprotein B; APOC2, apoprotein C2; APOE2, apoprotein E2; IDL, intermediate-density lipoprotein; LPL, lipoprotein lipase; VLDL, very low-density lipoprotein. 

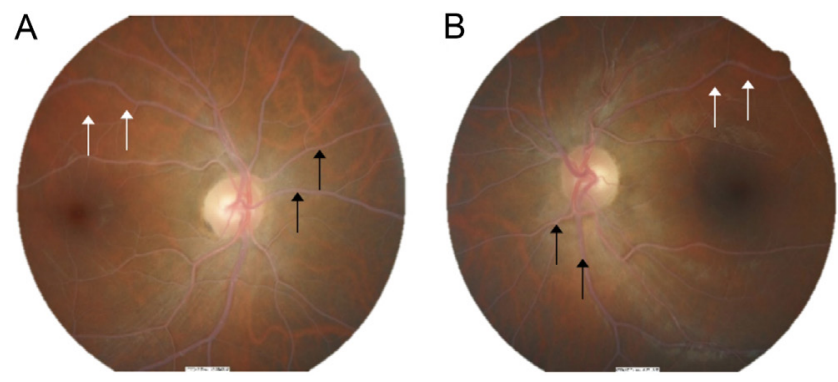

\section{Figure 1}

Retinal images of a patient's right (A) and left (B) eyes before treatment, showing normal optic discs with white retinal vessels (black arrows) in the posterior poles and peripheral retina. All the retinal vessels have a similar pale colour and are only distinguishable by size. The choroidal vessels have a similar pallor, giving the fundus a salmon-pink appearance (white arrows). These findings are suggestive of grade III lipaemia retinalis.

with recurrent pancreatitis. She had no significant family history. Her marriage was non-consanguineous, and she had five children, none of whom struggled with lipid abnormalities. She was a lifelong non-smoker and did not consume alcohol.

\section{Investigation}

Upon examination, the patient had multiple naevi on her skin, for which she was being managed conservatively by dermatologists. The rest of her examination was unremarkable. Laboratory investigations showed a raised triglyceride level of $57.7 \mathrm{mmol} / \mathrm{L}$ (reference range: $<2.0$ $\mathrm{mmol} / \mathrm{L}$ ) and total cholesterol level of $12.5 \mathrm{mmol} / \mathrm{L}$ (reference range: $<5.0 \mathrm{mmol} / \mathrm{L}$ ), markedly higher than previous readings. Her diabetes control was suboptimal, with an $\mathrm{HbA1c}$ of $74 \mathrm{mmol} / \mathrm{mol}$ (8.9\%). The remaining blood tests were within the normal limits. Following evaluation, the patient was diagnosed with grade III lipaemia retinalis, secondary to type I hyperlipidaemia.

\section{Treatment}

Following her diagnosis with severe hypertriglyceridemia and type I hyperlipidaemia, she was initially managed under a specialist multidisciplinary team (including diabetologist, lipid specialist and dietician) with various stand-alone and combination therapies with fibrates, cholestyramine, orlistat and statin over the years; however, these proved to be ineffective. Dietary interventions with dietician input consisting of fruits, vegetables, whole grains, legumes, nuts, fish, and low-fat dairy products rather than refined or processed foods, red meats, high concentrated sweets, eggs, and butter, strict glycaemic control, optimised with a combination of short- and long-acting insulin, omega-3 fish oil, and the use of medium-chain triglyceride oil led to some improvements in her lipid profile. The latter resulted in fat-soluble vitamin deficiency, confirmed by laboratory blood tests, which was subsequently treated with vitamins $\mathrm{D}$ and $\mathrm{E}$ supplementation following multidisciplinary team guidancen. She was also started on volanesorsen, which was well-tolerated, to lower the serum triglyceride level.. This lowered her serum triglycerides to single figures with no further admission with pancreatitis. However, there were some elevations of triglycerides depending on her glycaemic control as poor glycaemic control increases very low-density lipoprotein (VLDL) production.

\section{Outcome and follow-up}

Following significant changes, her lipid parameters improved, with a triglyceride level of $27.4 \mathrm{mmol} / \mathrm{L}$ and total cholesterol level of $7.6 \mathrm{mmol} / \mathrm{L}$ (Fig. 2; Supplementary materials, see section on supplementary materials given at the end of this article). These were further lowered to single figures $(7.5-10.5 \mathrm{mmol} / \mathrm{L})$ following introduction of volanesorsen. At the last follow-up, over 3 years after the first presentation, retinal images showed a complete resolution of lipaemia retinalis and normalisation of the retina (Fig. 3). She was genetically tested, and her family offered screening.

\section{Discussion}

Lipaemia retinalis is a rare manifestation of hyperlipidaemia and a direct consequence of elevated triglyceride and/or chylomicron levels in retinal vessels $(6,7,8)$. In secondary



Figure 2

Blood samples were collected at intervals over the case period when the patient consulted. The results show fluctuations in triglyceride (blue), and total cholesterol (orange) levels over time. Purple shaded area illustrates the period when the patient was diagnosed with diabetes mellitus. 

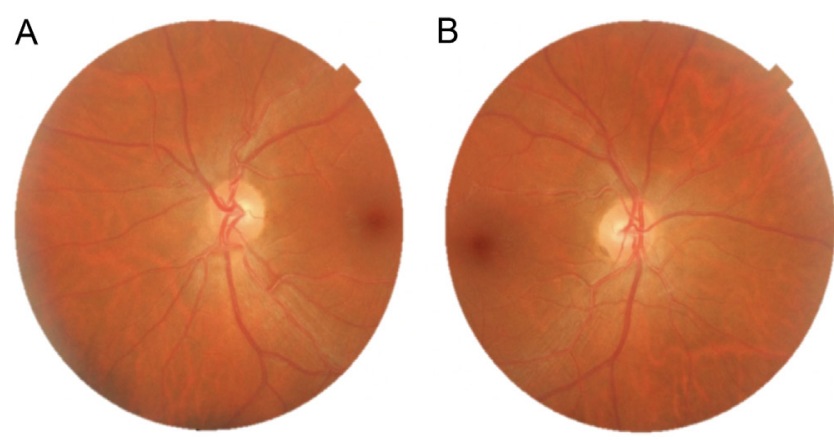

\section{Figure 3}

Retinal images of the patient's right $(A)$ and left $(B)$ eyes after treatment, showing a complete resolution of the ocular findings of lipaemia retinalis, with the retinal vessels and retina restored to their normal appearances.

hyperlipidaemia, a major cause of lipaemia retinalis is chylomicronaemia which results from uncontrolled diabetes mellitus (6). As for primary hyperlipidaemia, lipaemia retinalis is only observed in patients with type I, IV and $\mathrm{V}$ hyperlipidaemia without hypertriglyceridaemia, and is not commonly associated with lipaemia retinalis $(2,9)$.

The pathological appearance of lipaemia retinalis is due to the visualisation of triglyceride-laden chylomicrons circulating in the retinal vessels, which have a lightscattering effect and produce a turbid appearance $(3,6)$. As chylomicrons contain a high proportion of triglycerides (95\%), a raised triglyceride level is observed in lipaemia retinalis (6). Characteristic fundus changes, namely discolouration of the retinal vessels are primarily observed when plasma triglyceride levels are $>50 \mathrm{mmol} / \mathrm{L}$. This varies from a salmon-pink to creamy-white colour, depending on the plasma triglyceride level (6).

Early signs of lipaemia retinalis, at a triglyceride level of $50-90 \mathrm{mmol} / \mathrm{L}$, are confined to the peripheral retina, where the vessels appear thin and creamy (6). As the triglyceride level increases to $91-129 \mathrm{mmol} / \mathrm{L}$, vessels in the posterior pole become creamy as the lipaemia extends towards the optic disc (6). At a triglyceride level $>130 \mathrm{mmol} / \mathrm{L}$, retinal arteries and veins adopt a similar creamy-white appearance and are only distinguishable by size (6). Similar changes in

Table 2 Clinical features of lipaemia retinalis (9). To view the full dataset for the blood results within the ScholarOne system please click the 'Files' tab to download the documents.

\begin{tabular}{|c|c|c|}
\hline Grade & Intensity & Clinical appearance \\
\hline 1 & Early & White and creamy peripheral vessels \\
\hline II & Moderate & $\begin{array}{l}\text { Creamy-coloured vessels extending } \\
\text { towards optic disc }\end{array}$ \\
\hline III & Marked & $\begin{array}{l}\text { Salmon-coloured retina, all vessels having } \\
\text { milky aspect }\end{array}$ \\
\hline
\end{tabular}

the choroidal vessels gives the fundus a salmon-coloured appearance (6).

A grading system describes the stages of lipaemia retinalis (Table 2), of which grade III has rarely been described $(6,9)$. Based on this classification, our patient had grade III changes. This is particularly interesting as our patient had a relatively low triglyceride level of 57.7 $\mathrm{mmol} / \mathrm{L}$. It must be noted that not all patients with elevated triglyceride or chylomicron levels show lipaemia retinalis, suggesting other influencing factors, such as haematocrit and translucency of vessels (9). We also considered the impact of ethnicity as Asian populations have been shown to have more severe manifestations of metabolic syndrome (10). More studies are needed to confirm this, perhaps, with a new grading system specifically for Asian populations.

Vision is initially normal, but may be affected by advanced lipaemia or in the presence of age-related macular degeneration (6). However, persistent lipaemia retinalis can lead to extensive, irreversible vision loss (6). Hence, rapid recognition and reversal are needed and have been shown to improve prognosis (6). A low-fat diet can be used for management as well as primary and secondary prevention of lipaemia retinalis in high-risk individuals (11). Currently, there are no specific medications that can be used to treat lipaemia retinalis. Lipid-lowering therapies with fibrates, nicotinic acid and n-3 polyunsaturated fatty acid can reduce triglycerides levels by up to $50 \%$, which may cause rapid reversal of abnormal findings $(12,13$, 14). Volanesorsen, a new pharmacological drug received by our patient, , inhibits hepatic APOC3 mRNA and has been shown to significantly reduce triglyceride levels. APOC3 glycoprotein is synthesised in the liver and to a lesser extent in the small intestine and has recently being recognised as a key regulator of plasma triglyceride levels. APOC3 is a potent inhibitor of lipoprotein lipase resulting in inhibition of lipolysis, hence reducing triglyceride levels. It has also been reported to inhibit hepatic lipase activity, promoting VLDL production and secretion, as well as inhibiting the clearance of triglyceride-rich lipoproteins (15). Injection site reactions and thrombocytopaenia were the most commonly reported adverse events of volanesorsen - fortunately, this was well-tolerated by our patient. Improvement of the lipid profile might also prevent acute complications of hypertriglyceridemia, such as acute pancreatitis and cardiovascular events. Optimisation of glycaemic control in patients with poorlycontrolled diabetes is essential for diabetes management (16). As in this case, optimal management lowered the plasma triglyceride levels and lead to a complete resolution of fundoscopic findings alongside the other signs and 
symptoms of chylomicronaemia syndrome $(4,6,7)$. This highlights the importance of involving dieticians and wider multidisciplinary team as our patient was initially resistant to conventional lipid-lowering agents. Earlier dietician input could have prevented both chronic pancreatitis and the development of lipaemia retinalis. Exchange transfusion has been proposed as a management option for patients with severe hypertriglyceridaemia (17, 18). Furthermore, surgical management such as ileal bypass surgery has also been shown to be effective in improving the lipid profile. However, surgical interventions should only be opted for in cases where the patient does not respond to conventional medical therapies.

In the UK, the National Institute for Health and Care Excellence provides a clear guidance for genetic testing, identification and management of familial hypercholesterolemia (19). This includes a structured annual review for any symptoms of coronary heart disease, smoking status, fasting lipid profile and current management options with no specific emphasis on signs and symptoms of lipaemia retinalis. Given the potential visual deficits and systemic complications of lipaemia retinalis and severe hypertriglyceridemia, we suggest regular screening for lipaemia retinalis in all patients with familial hypercholesterolaemia to prevent morbidity and mortality from this condition.

\section{Conclusion}

Lipaemia retinalis is an initially asymptomatic condition, which is an important clinical indicator, with high specificity, for elevated triglyceride and chylomicron levels. Because it does not typically cause visual symptoms and presents first in the peripheral retina, lipaemia retinalis is underdiagnosed. It can be rapidly resolved by lipidlowering measures; however, persistent lipaemia retinalis and hypertriglyceridaemia may lead to irreversible visual deficits and more severe systemic complications. Given that lipaemia retinalis is an important ocular finding of potential cardiovascular disease, it is essential that clinicians recognise the signs and symptoms so that treatment can be rapidly commenced.

\section{Supplementary materials}

This is linked to the online version of the paper at https://doi.org/10.1530/ EDM-21-0051.

\section{Declaration of interest}

The authors declare that there is no conflict of interest that could be perceived as prejudicing the impartiality of the research reported.

\section{Funding}

This study did not receive any specific grant from any funding agency in the public, commercial or not-for-profit sector.

\section{Patient consent}

Written informed consent has been obtained from the patient (or patient's guardian) for publication of the submitted article and accompanying images.

\section{References}

1 Ginsberg HN. Diabetic dyslipidemia: basic mechanisms underlying the common hypertriglyceridemia and low HDL cholesterol levels. Diabetes 199645 (Supplement 3) S27-S30. (https://doi.org/10.2337/ diab.45.3.s27)

2 Heyl AG. Intra-ocular lipaemia. Transactions of the American Ophthalmological Society 18803 54-66.

3 Rymarz E, Matysik-Wozniak A, Baltaziak L, Prystupa A, Sak J \& Grzybowski A. Lipemia retinalis - an unusual cause of visual acuity deterioration. Medical Science Monitor 201218 CS72-CS75. (https://doi. org/10.12659/msm.883257)

4 Trese MGJ, Price JM \& Bohra L. Lipemia retinalis as the presenting sign of undiagnosed type 1 diabetes mellitus resulting in severe secondary hypertriglyceridemia. Journal of AAPOS 201822 405-407. (https://doi. org/10.1016/j.jaapos.2018.03.014)

5 Fredrickson DS \& Lees RS. A system for phenotyping hyperlipoproteinemia. Circulation 196531 321-327. (https://doi. org/10.1161/01.cir.31.3.321)

6 Rayner S, Lee N, Leslie D \& Thompson G. Lipaemia retinalis: a question of chylomicrons? Eye 199610 603-608. (https://doi. org/10.1038/eye.1996.138)

7 Brunzell JD \& Bierman EL. Chylomicronaemia syndrome: interaction of genetic and acquired hypertriglyceridaemia. Medical Clinics of North America 198266 455-468. (https://doi.org/10.1016/S00257125(16)31430-4)

8 Chaudhury D, Meenakshi R, Ramakrishnan R, Mitra A \& Raju S. Lipaemia retinalis. Indian Journal of Ophthalmology 201526 107-110.

9 Vinger PF \& Sachs BA. Ocular manifestations of hyperlipoproteinaemia. American Journal of Ophthalmology 197070 563-573. (https://doi.org/10.1016/0002-9394(70)90890-1)

10 Palaniappan LP, Wong EC, Shin JJ, Fortmann SP \& Lauderdale DS. Asian Americans have greater prevalence of metabolic syndrome despite lower body mass index. International Journal of Obesity 201135 393-400. (https://doi.org/10.1038/ijo.2010.152)

11 Yin HY, Warman R, Suh EH \& Cheng AM. Exceptionally elevated triglyceride in severe lipemia retinalis. International Medical Case Reports Journal 20169 333-336. (https://doi.org/10.2147/IMCRJ. S118594)

12 Stalenhoef AF, de Graaf J, Wittekoek ME, Bredie SJ, Demacker PN $\&$ Kastelein JJ. The effect of concentrated n-3 fatty acids versus gemfibrozil on plasma lipoproteins, low density lipoprotein heterogeneity and oxidizability in patients with hypertriglyceridemia. Atherosclerosis 2000153 129-138. (https://doi.org/10.1016/s00219150(00)00381-6)

13 Chapman MJ. Fibrates in 2003: therapeutic action in atherogenic dyslipidaemia and future perspectives. Atherosclerosis 2003 171 1-13. (https://doi.org/10.1016/s0021-9150(03)00156-4)

14 Harris WS, Pottala JV, Thiselton DL, Varvel SA, Baedke AM, Dayspring TD, Warnick GR \& McConnell JP. Does APOE genotype modify the relations between serum lipid and erythrocyte omega-3 fatty acid levels? Journal of Cardiovascular Translational Research 20147 526-532. (https://doi.org/10.1007/s12265-014-9554-8) 
15 Witztum JL, Gaudet D, Freedman SD, Alexander VJ, Digenio A, Williams KR, Yang Q, Hughes SG, Geary RS, Arca M, et al. Volanesorsen and triglyceride levels in familial chylomicronemia syndrome. New England Journal of Medicine 2019381 531-542. (https://doi. org/10.1056/NEJMoa1715944)

16 Leaf DA. Chylomicronemia and the chylomicronemia syndrome: a practical approach to management. American Journal of Medicine 2008 121 10-12. (https://doi.org/10.1016/j.amjmed.2007.10.004)

17 Capitena CE, Wagoner HJ, Ruzas CM, Bennett TD, Baker PR, Jung JL $\&$ Weisfeld-Adams JD. Rapid resolution of infantile lipemia retinalis following exchange transfusion. Journal of Inherited Metabolic Disease 201639 889-890. (https://doi.org/10.1007/s10545-016-9968-3)

18 Schaap-Fogler M, Schurr D, Schaap T, Leitersdorf E \& Rund D. Longterm plasma exchange for severe refractory hypertriglyceridemia: a decade of experience demonstrates safety and efficacy. Journal of Clinical Apheresis 200924 254-258. (https://doi.org/10.1002/ jca.20224)

19 National Institute for Health and Clinical Care Excellence. Familial hypercholesterolaemia: identification and management, 2008. (available at: https://www.nice.org.uk/guidance/cg71)

Received in final form 6 August 2021

Accepted 29 September 2021 